\title{
Correction: Integrative network biology analysis identifies miR-508- $3 p$ as the determinant for the mesenchymal identity and a strong prognostic biomarker of ovarian cancer
}

\author{
Linjie Zhao ${ }^{1} \cdot$ Wei Wang ${ }^{2} \cdot$ Lian Xu $\cdot$ Tao $\mathrm{Yi}^{1} \cdot \mathrm{Xia}_{\mathrm{Zhao}}{ }^{1} \cdot \mathrm{Yuquan}_{\mathrm{Wei}}{ }^{1} \cdot$ Louis Vermeulen $\left(\mathbb{D} \cdot\right.$ Ajay Goel $^{\cdot}$ \\ Shengtao Zhou ${ }^{1} \cdot$ Xin Wang ${ }^{2}{ }^{2}$
}

Published online: 3 September 2019

(c) The Author(s) 2019

\section{Correction to: Oncogene}

https://doi.org/10.1038/s41388-018-0577-5

published online 26 November 2018

Following publication of this article, the authors noted that they accidentally misplaced the figure in supplementary
Fig. $7 \mathrm{~b}$ when re-editing the figures to meet the format and the image resolution of the journal. The amended version of supplementary Fig. 7 is provided below. This change does not affect the results or conclusions reported in the paper.

These authors contributed equally: Linjie Zhao, Wei Wang, Lian Xu

The original article can be found online at https://doi.org/10.1038/ s41388-018-0577-5.

Shengtao Zhou

taotaovip2005@163.com

$\triangle$ Xin Wang

xin.wang@cityu.edu.hk

1 Department of Obstetrics and Gynecology, Key Laboratory of Birth Defects and Related Diseases of Women and Children of MOE and State Key Laboratory of Biotherapy, West China Second University Hospital, Sichuan University and Collaborative Innovation Center, Chengdu, China

2 Department of Biomedical Sciences, City University of Hong Kong, Kowloon Tong, Hong Kong 
a

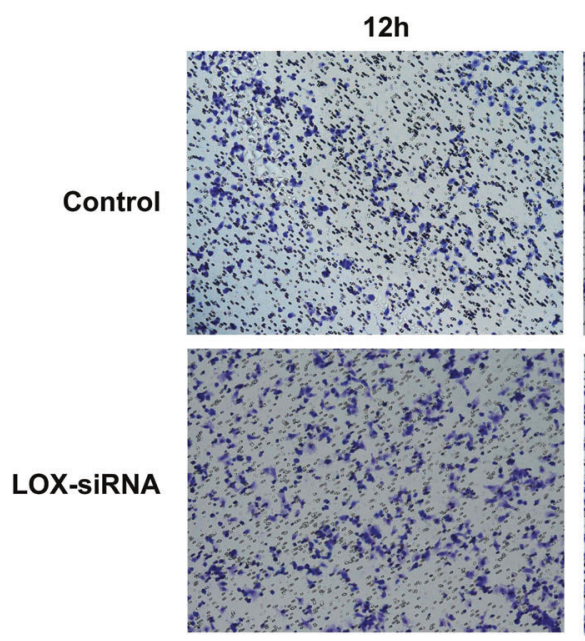

miR-508-3p inhibitor-transfected OV56 cells

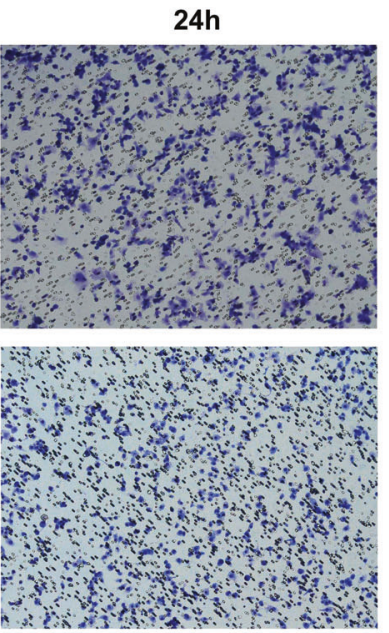

b

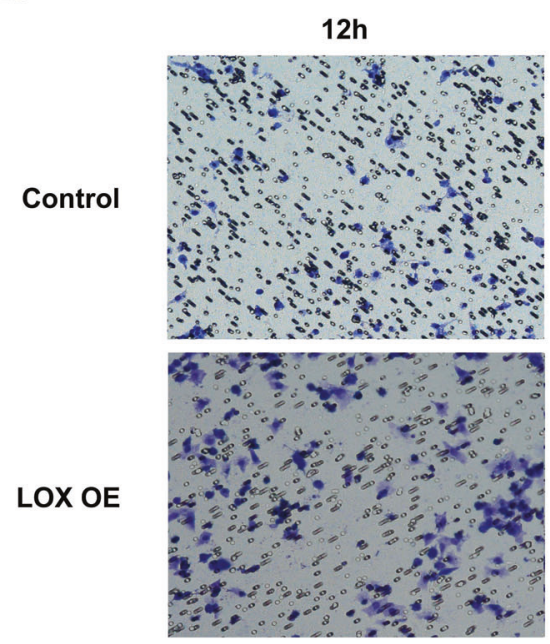

Fig. S7 a Transwell chamber analysis of control or LOX silenced OV56 cells transfected with miR-508-3p inhibitor. b Transwell chamber analysis of control or LOX-overexpressing COV504 cells
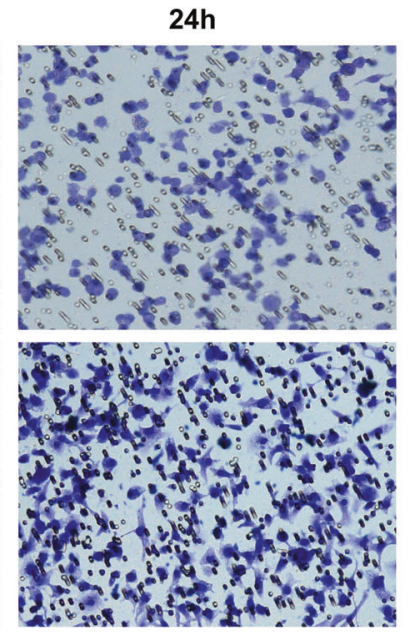

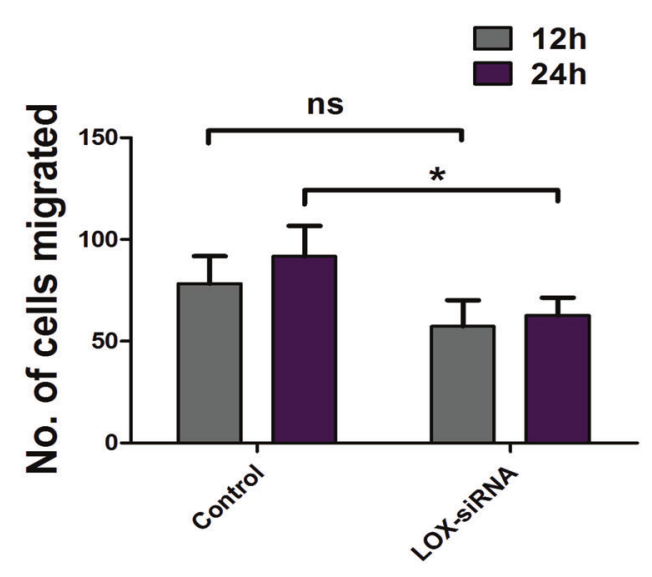

transfected with miR-508-3p mimic. In all bar plots, $p$-values are based on two-tailed Student's t-tests $(* P<0.05, * * P<0.01, * * * P<0.001)$ 